1 Department of Pediatrics, Faculty of Medical Sciences, University of Campinas, 13081-970, P.O. Box: 6111 . Campinas, São Paulo, Brazi

${ }^{2}$ Department of Medical Genetics and Genomic Medicine, Faculty of Medical Sciences, University of Campinas, 13081-970, P.O. Box: 6111, Campinas, São Paulo, Brazil

${ }^{3}$ Center for Research in Pediatrics, Faculty of Medical Sciences, University of Campinas, 13081-970, P.O. Box: 6111, Campinas, São Paulo, Brazil

‘Boldrini Children's Center, Campinas, São Paulo, Brazil

${ }^{5}$ Department of Clinical Pathology, Faculty of Medical Sciences, University of Campinas, 13081-970, P.O. Box: 6111, Campinas, São Paulo, Brazil

Conflicts of interest: All the authors declare that they have no conflicts of interest.

Corresponding author:

[FALM] Department of Pediatrics and Department of Medical Genetics and Genomic Medicine, Faculty of Medical Sciences, University of Campinas. Rua Tessália Vieira de Camargo, 126, Cidade Universitária Zeferino Vaz, Barão Geraldo, Campinas, São Paulo, Brazil. E-mail: fernandolimamarson@hotmail.com; Phone: +55 1935218902 , FAX: +55 1935218907. [CEL] Department of Clinical Pathology, Faculty of Medica Sciences, University of Campinas. Rua Tessália Vieira de Camargo, 126 Cidade Universitária Zeferino Vaz, Barão Geraldo, Campinas, São Paulo, Brazil. E-mail: levy.emilio@gmail.com; Phone: +551935219446 ;

FAX: +551935217322

Received: January 24, 2018

Accepted: October 09, 2018

\section{Oral health in patients with cystic fibrosis}

\author{
Aline Cristina Gonçalves ${ }^{1}$, Fernando Augusto Lima \\ Marson 1,2,3, Regina Maria Holanda Mendonça ${ }^{4}$, \\ José Dirceu Ribeiro ${ }^{1}$, Antônio Fernando Ribeiro ${ }^{1}$, \\ Carlos Emílio Levy ${ }^{5, a}$
}

The oral health in patients with cystic fibrosis (CF) is not well studied. Aim: Our aim was to describe the frequency of dental problems and to perform the description of plaque index and gingival index in patients with CF. Methods: We performed a descriptive study about oral health enrolling 94 patients with CF. Results: The CF diagnosis was done considering the sweat test with chloride values $\geq 60 \mathrm{mmol} / \mathrm{L}$. The permanent dentition was predominant in $57 / 94(60.64 \%)$ patients with CF - 28/57 (49.12\%) caries, 57/57 (100\%) filled teeth and 1/57 $(1.75 \%)$ missing tooth. Deciduous teeth occurred in $37 / 94$ (39.36\%) patients with CF - 22/37 (59.46\%) carious, 29/37 (78.38\%) exfoliated teeth, $20 / 37$ (54.05\%) filled teeth. Also, the plaque index and gingival index had scored less than one in many cases ( $\geq 50 \%$ of the cases). Conclusions: Patients with CF showed oral health problems with prevalence comparable to that of the normal population and low values in the plaque index and gingival index.

Keywords: Cystic fibrosis. Dental caries. Dental plaque index. Periodontal index. Oral health. 


\section{Introduction}

A high prevalence of enamel defects is seen in cystic fibrosis (CF) (OMIM: \#219700), and it is associated with the disease severity (metabolic and nutritional disorders) and its chronic therapy ${ }^{1,2}$. Also, patients with CF have higher prevalence of dental calculus, probably due to the high saliva calcium and phosphate associated with the disease, contributing to tartar formation ${ }^{3}$. However, caries occurs at lower to equal rates in patients with CF than healthy controls subjects ${ }^{4,5}$. Long-term use of antibiotics and pancreatic enzymes could protect the patients against caries. Finally, the presence of enamel defects can be classified as opacity (qualitative defect: white or discolored enamel with a smooth surface and normal thickness) and hypoplasia (quantitative defect: pits or rows of pits, grooves, partial or complete absence of enamel) and should be studied in patients with $\mathrm{CF}^{2,6}$. In this context, our primary objective (outcome) was investigating the oral health from patients with CF and the secondary objective (outcome) was verify the values of plaque index and gingival index.

\section{Materials and methods}

A descriptive study was carried out on 94 patients with CF (Chloride $\geq 60 \mathrm{mmol} / \mathrm{L}$ ) from University of Campinas. The sweat test is the gold standard for the CF diagnosis. The interpretation of the sweat test should be concomitant to the clinical context, and when possible, molecular analysis of the CFTR gene. For the CF diagnosis it is necessary to perform at least two sweat tests with a chloride concentration $\geq 60 \mathrm{mmol} / \mathrm{L}$. When the value is $<30 \mathrm{mmol} / \mathrm{L}$, the CF diagnosis is considered unlikely. Concentrations of chloride in sweat with values between $\geq 30$ and $<60 \mathrm{mmol} / \mathrm{L}$ are considered doubtful and should be repeated 7,8 .

Dental plaque accumulation, caries and dental enamel defects were examined to determine the oral health in patients with CF. Also, a baseline score of the oral hygiene was done using the plaque index of Silness and $\operatorname{Loe}^{9}(1964)$ and the gingival health using the gingival index of Sillness and Loe (1964).

Plaque index was assigned as follow: 0 - no plaque; 1 - a film of plaque adhering to the free gingival margin and adjacent area of the tooth. The plaque may be seen in situ only after application of disclosing solution or by using the probe on the tooth surface; 2- moderate accumulation of soft deposits within the gingival pocket, or the tooth and gingival margin which can be seen with the naked eye; 3-abundance of soft matter within the gingival pocket and/or on the tooth and gingival margin.

Gingival index was assigned as follow: 0- normal gingiva; 1- mild inflammation - slight change in color and slight edema but no bleeding on probing; 2- moderate inflammation - redness, edema and glazing, bleeding on probing; 3 - severe inflammation - marked redness and edema, ulceration with tendency to spontaneous bleeding ${ }^{10}$.

Use of dornase alfa (recombinant human deoxyribonuclease I) and antibiotics was obtained from patients' healthcare records. Chronic use of antibiotics was defined as the use of antibiotics for at least two years in last five years.

The study was approved by Ethics Committee of the University of Campinas (\#157/2010). 
Table 1. Plaque index, gingival index and drug used in patients with cystic fibrosis.

\begin{tabular}{lcc}
\hline Score & Plaque index $-\mathbf{n}(\%)$ & Gingival index - $\mathbf{n}(\%)$ \\
\hline 0 & $24(25.53)$ & $31(32.98)$ \\
\hline 1 & $38(40.42)$ & $40(42.55)$ \\
\hline 2 & $26(27.66)$ & $18(19.15)$ \\
\hline 3 & $6(6.39)$ & $5(5.32)$ \\
\hline Drugs & $32(34.04 \%)$ \\
\hline Dorntibiotic + dornase alfa & $8(8.51 \%)$ \\
\hline Antibiotics only & $22(23.4 \%)$ \\
\hline No medication & $32(34.04 \%)$ \\
\hline
\end{tabular}

The baseline score of the oral hygiene was done using the plaque index of Loe and Sillness, and the gingival health using the gingival index of Loe and Sillness (10). Plaque index: 0 - no plaque; 1 - a film of plaque adhering to the free gingival margin and adjacent area of the tooth; 2- moderate accumulation of soft deposits within the gingival pocket, or the tooth and gingival margin which can be seen with the naked eye; 3 - abundance of soft matter within the gingival pocket and/or on the tooth and gingival margin. Gingival index: 0 - normal gingiva; 1- mild inflammation - slight change in color and slight edema but no bleeding on probing; 2- moderate inflammation - redness, edema and glazing, bleeding on probing; 3- severe inflammation - marked redness and edema, ulceration with tendency to spontaneous bleeding.

\section{Results}

In our sample, we included 94 patients with CF with mean age of 12.74 years, and 45/94 (47.87\%) were male.

The permanent teeth dentition was more frequent in 57/94 (60.64\%) patients: $28 / 57$ (49.12\%, mean of 0.50 lesions/patient) had caries, $57 / 57$ filled teeth $(100 \%$, mean of 1 tooth/patient) and only $1 / 57$ patient (1.75\%) had a missing tooth. Deciduous teeth occurred in 37/94 (39.36\%) patients: 22/37 (59.46\%, mean of 0.59/patient) had carious, $29 / 37$ (78.38\%, mean of 0.78 /patient) had exfoliated teeth and $20 / 37$ (54.05\%, mean of 0.54 /patient) had filled teeth. In our casuistic, we observed few $(36.57 \%)$ defects in the dental enamel. Finally, regarding the oral health in patients with $\mathrm{CF}$, it was observed a higher percentage of caries in the permanent dentition, while the defects of the enamel were more prevalent in the primary dentition.

Plaque index and gingival index are shown in Table 1, and both indexes had a scored less than one in many cases ( $\geq 50 \%$ of the cases). Also, the drugs used were set in the same table.

\section{Discussion}

We need to treat the patients with $\mathrm{CF}$, taking care of their oral health equates taking care of their other problems related to CF disease ${ }^{11}$. In this context, we performed the first study regarding the oral health in patients with CF from Brazil.

While children with CF may be at lower risk for dental caries, adolescents with CF may not be at lower caries than those without $\mathrm{CF}^{12-17}$. This fact can be associated with the use of some antibiotics, which targets Pseudomonas aeruginosa, and do not affect the Streptococcus mutans. Although there are no studies about this issue, one hypoth- 
esis is that although the antibiotic does not affect $S$. mutans, the continuous use this medicament alters the oral microbiome and consecutively, the components from oral cavity $^{18}$. Therefore, adolescents with CF would lose the benefits of caries protection afforded in childhood ${ }^{19}$.

Moreover, in a study to identify the relationship between the number of caries and the S. mutans and lactobacilli, a lower amount of caries occurred in p.Phe508del homozygotes when compared to heterozygous patient ${ }^{20}$. Also, previously, a study compared patients with CF and healthy control subjects and no difference was observed regarding the number of caries ${ }^{21}$. In this context, the Cystic fibrosis transmembrane conductance regulator (CFTR) genotype should be considered to evaluate the oral health in patients with CF because the pathogenic variants in CFTR gene can alter the components from oral cavity, and consecutively, the prevalence of some bacteria. Also, severe CFTR genotypes are a risk group to use wide spectrum and aggressive antibiotic therapy causing alteration in the human microbiome, including the oral cavity microbiome.

The development of caries is a complex process related to environmental and hereditary factors. The low prevalence of caries in patients with CF, observed in some studies, can be attributed to several factors. Maybe, the effects of $\mathrm{pH}$ by consuming a diet with a high content of dairy products protect the teething from deterioration ${ }^{1-3,19}$. Also, the reduced amount of caries in CF patients could be related to a higher $\mathrm{pH}$ and storage capacity of saliva ${ }^{22}$.In this way, the incorporation of salivary calcium and phosphate into the enamel may be a post-eruption maturation process that gives the tooth greater resistance to demineralization. The CFTR protein is expressed in the salivary glands, but its effects on salivary gland and oral health, including caries, are inconsistent.

The results regarding caries are controversial and in literature the following information can be identified: (i) no association between salivary flow rate or buffering capacity and caries prevalence; (ii) negative association between salivary $\mathrm{pH}$ and caries prevalence, but this association was no longer significant after adjusting for age; (iii) no significant interaction between salivary flow rate and buffering capacity or between antibiotic use and the salivary factors. This issued should be studied at CF disease, because at CF we had ionic alterations at saliva and in salivary glands functions, causing an abnormal ionic environmental at oral cavity. Also, we had the chronic use of antibiotic therapy promoting a "chronic" adaptation from oral cavity microbiome regarding the CF treatment.

In this context, as previously discussed, the patients with CF make use of antibiotics due to chronic lung infection and this fact could reduce the prevalence of caries, since it alters the oral microbiome ${ }^{15,16}$. Azithromycin is often prescribed and it affects gram-positive bacteria. Also, it penetrates dental biofilm, with good absorption in periodontal tissue, and can be retained in the periodontal pocket for up to 14 days ${ }^{23}$. Moreover, patients with CF and their parents tend to be motivated to health care and therefore, in general, show adherence to dental treatment. In addition, the majority of patients with CF use pancreatic enzyme replacement, that reduce the incidence of caries ${ }^{24}$, although our study was not comparative we observed a low frequency of caries lesions. 
In addition, changes in salivary $\mathrm{pH}$, oxidation-reduction reactions or continuous use of antibiotics may cause imbalance in the oral microbiome with increase of pathogenic or potentially pathogenic bacteria, triggering diseases that may be a non-serious condition such as gingivitis or caries, or being severe and give rise to infections 25,26 .

In CF, it is possible that the CFTR gene mutations that causes the disease could be responsible for the high incidence of enamel defects ${ }^{27}$. Molecular studies have shown that the CFTR gene is expressed in developing teeth and other mineralized tissues. The CFTR gene mutations cause an alteration in the $\mathrm{pH}$ during enamel development, which results in a lack of calcium influx during enamel maturation, hypomineralization, and alteration of the normal crystal growth and of the protein processing functions necessary for optimal enamel formation ${ }^{28,29}$.

Abnormal enamel mineralization, ion concentrations and molecular evidence of CFTR mRNA expression by odontogenic cells strongly suggest that CFTR plays an important role in enamel formation 2,29 . Although our study demonstrated a low incidence of enamel defects and opacities, the absence of a group of healthy subjects (control) prevents a comparison with the general population without CF.

Enamel and dentin have chemical components that participate in ion exchanges and demineralization and remineralization processe ${ }^{30}$. Since CFTR is present in teeth and CF is basically characterized by ion transport defect and ion channels are related to the biological functions of dental development, perhaps this explains a considerable frequency of tooth enamel defects in our sample.

Finally, Azevedo et al. (2006) evaluated patients with CF and showed that more than $90 \%$ of subjects had at least one enamel defect (demarcated opacities, diffuse opacities and enamel hypoplasia) $)^{31}$. The previous prevalence was greater than the observed in our data sample ( 40\%).

In conclusion, patients with CF showed oral health problems with prevalence comparable to that of the normal population. Also, dental plaque accumulation, caries and dental enamel defects were examined and presented problems similar to healthy control subjects from other studies. In addition, plaque index and gingival index had low values. Future studies should investigate cariogenic bacteria levels, salivary conditions, medication use and CFTR genotype to give a better conclusion about health condition at oral cavity in patients with CF.

\section{References}

1. Ferrazzano GF, Orlando S, Sangianantoni G, Cantile T, Ingenito A. Dental and periodontal health status in children affected by cystic fibrosis in a southern Italian region. Eur J Paediatr Dent. 2009 Jun;10(2):65-8.

2. Ferrazzano GF, Sangianantoni G, Cantile T, Amato I, Orlando S, Ingenito A. Dental enamel defects in Italian children with cystic fibrosis: an observational study. Community Dent Health. 2012 Mar;29(1):106-9

3. Narang A, Maguire A, Nunn JH, Bush A. Oral health and related factors in cystic fibrosis and other chronic respiratory disorders. Arch Dis Child. 2003 Aug;88(8):702-7. 
4. Peker S, Kargul B, Tanboga I, Tunali-Akbay T, Yarat A, Karakoc F, et al. Oral health and related factors in a group of children with cystic fibrosis in Istanbul, Turkey. Niger J Clin Pract. 2015 Jan-Feb;18(1):56-60. doi: 10.4103/1119-3077.146980.

5. Sarvas EW, Huebner CE, Scott JM, Aps JK, Chi DL. Dental utilization for Medicaid-enrolled children with cystic fibrosis. Spec Care Dentist. 2016 Nov;36(6):315-320. doi: 10.1111/scd.12193.

6. Clarkson J, O'Mullane D. A modified DDE Index for use in epidemiological studies of enamel defects. J Dent Res. 1989 Mar;68(3):445-50.

7. Faria AG, Marson FAL, Gomez CCS, Servidoni MF, Ribeiro AF, Ribeiro JD. Thirty Years of Sweat Chloride Testing at One Referral Center. Front Pediatr. 2017 Oct 26;5:222. doi: 10.3389/fped.2017.00222.

8. Farrell PM, White TB, Ren CL, Hempstead SE, Accurso F, Derichs N, et al. Diagnosis of Cystic Fibrosis: Consensus Guidelines from the Cystic Fibrosis Foundation. J Pediatr. 2017 Feb;181S:S4-S15.e1. doi: 10.1016/j.jpeds.2016.09.064.

9. Silness J, Loe H. Periodontal disease in pregnancy. ii. correlation between oral hygiene and periodontal condtion. Acta Odontol Scand. 1964 Feb;22:121-35.

10. Löe H. The Gingival Index, the Plaque Index and the Retention Index Systems. J Periodontol. 1967 Nov-Dec;38(6):Suppl:610-6.

11. Harrington N, Barry PJ, Barry SM. Dental treatment for people with cystic fibrosis. Eur Arch Paediatr Dent. 2016 Jun;17(3):195-203. doi: 10.1007/s40368-016-0229-9.

12. Primosch RE, Brearley L. Dental caries experience in patients with cystic fibrosis. J Dent Res. 197888,57:150.

13. Primosch RE. Tetracycline discoloration, enamel defects, and dental caries in patients with cystic fibrosis. Oral Surg Oral Med Oral Pathol. 1980 Oct;50(4):301-8.

14. Kinirons MJ. Dental health of patients suffering from cystic fibrosis in Northern Ireland. Community Dent Health. 1989 Jun;6(2):113-20.

15. Aps JK, Van Maele GO, Martens LC. Caries experience and oral cleanliness in cystic fibrosis homozygotes and heterozygotes. Oral Surg Oral Med Oral Pathol Oral Radiol Endod. 2002 May;93(5):560-3.

16. Chi DL. Dental caries prevalence in children and adolescents with cystic fibrosis: a qualitative systematic review and recommendations for future research. Int J Paediatr Dent. 2013 Sep;23(5):376-86. doi: 10.1111/ipd.12042.

17. O'Keefe E. Are children and adolescents with cystic fibrosis at lower risk of caries? Evid Based Dent. 2014 Jun;15(2):46-7. doi: 10.1038/sj.ebd.6401024.

18. Jagels AE, Sweeney EA. Oral health of patients with cystic fibrosis and their siblings. J Dent Res. 1976 Nov-Dec;55(6):991-6.

19. Peker S, Mete S, Gokdemir Y, Karadag B, Kargul B. Related factors of dental caries and molar incisor hypomineralisation in a group of children with cystic fibrosis. Eur Arch Paediatr Dent. 2014 Aug;15(4):275-80. doi: 10.1007/s40368-014-0112-5.

20. Aps JK, Van Maele GO, Claeys G, Martens LC. Mutans streptococci, lactobacilli and caries experience in cystic fibrosis homozygotes, heterozygotes and healthy controls. Caries Res. 2001 Nov-Dec;35(6):407-11.

21. Martens LC, Aps JKM, Van Maele GOG. Is oral health at risk in people with cystic fibrosis? Eur J Paediat Dent. 2001 Mar;2:21-7.

22. Kinirons MJ. Increased salivary buffering in association with a low caries experience in children suffering from cystic fibrosis. J Dent Res. 1983 Jul;62(7):815-7. 
23. Hirsch R. Periodontal healing and bone regeneration in response to azithromycin. Aust Dent J. 2010 Jun;55(2):193-9. doi: 10.1111/j.1834-7819.2010.01227.x.

24. Sweeney EA, Shaw JH. The effect of dietary pancreatin supplements on dental caries and on the composition of saliva in caries-susceptible rats. J Dent Res. 1965 Sep-0ct;44(5):973-6.

25. Faran Ali SM, Tanwir F. Oral microbial habitat a dynamic entity. J Oral Biol Craniofac Res. 2012 Sep-Dec;2(3):181-7. doi: 10.1016/j.jobcr.2012.07.001.

26. Marques SC, Rezende JDGOS, Alves LADF, Silva BC, Alves E, De Abreu LR, et al. Formation of biofilms by Staphylococcus aureus on stainless steel and glass surfaces and its resistance to some selected chemical sanitizers. Bra J Microbiol. $2007 \mathrm{Jul} / \mathrm{Sep} ; 38(3): 538-43$. doi: 10.1590/S1517-83822007000300029.

27. Sui W, Boyd C, Wright JT. Altered pH regulation during enamel development in the cystic fibrosis mouse incisor. J Dent Res. 2003 May;82(5):388-92.

28. Wright JT, Kiefer $\mathrm{CL}$, Hall $\mathrm{KI}$, Grubb BR. Abnormal enamel development in a cystic fibrosis transgenic mouse model. J Dent Res. 1996 Apr;75(4):966-73.

29. Arquitt CK, Boyd C, Wright JT. Cystic fibrosis transmembrane regulator gene (CFTR) is associated with abnormal enamel formation. J Dent Res. 2002 Jul;81(7):492-6.

30. Duan X. Ion channels, channelopathies, and tooth formation. J Dent Res. 2014 Feb;93(2):117-25. doi: $10.1177 / 0022034513507066$.

31. Azevedo TD, Feijó GC, Bezerra AC. Presence of developmental defects of enamel in cystic fibrosis patients. J Dent Child (Chic). 2006 Sep-Dec;73(3):159-63. 